\title{
Macrobe et les âmes héroïques (Rabelais, Quart Livre, chapitres 25 à 28)
}

\author{
DIANE DESROSIERS-BONIN
}

Au sortir de la Tempête en mer, Pantagruel et ses compagnons en quête du mot de la Dive Bouteille font escale à l'île des Macréons. Les insulaires et le "vieil Macrobe" les accueillent. L'édition partielle du Quart Livre publiée en 1548 s'achève sur cette rencontre. Par ailleurs, l'édition complète de $1552^{1}$ donne suite au récit et développe non seulement ce qui nous semble le thème central des chapitres 25 à 28 mais aussi une problématique de tout premier intérêt à la Renaissance: le salut de l'homme lié à l'exercice des vertus civiles.

Dans ce contexte, le recours au nom de $\mathrm{Macrobe}^{2}$ se révèle particulièrement suggestif. ${ }^{3}$ En effet, ce personnage pourrait évoquer un autre Macrobe, ${ }^{4}$ écrivain latin du IVe siècle, auteur des Saturnales et surtout commentateur du Songe de Scipion. Ce commentaire, célèbre tout au long du moyen âge et de la Renaissance, avait assuré la transmission du seul fragment connu du De republica de Cicéron. Roger Bacon et Pétrarque avaient vainement cherché le manuscrit complet de ce traité que l'on croyait perdu ou, selon l'intuition de Pierre Ramus, caché. ${ }^{5}$

Pendant des siècles, on ne connut donc du De republica que le sixième et dernier livre commenté par Macrobius. Si, grâce surtout aux travaux de Schedler et de Stahl, ${ }^{6}$ on a pu apprécier l'influence de Macrobius au moyen âge, la fortune dont il jouit à la Renaissance demeure encore aujourd'hui très peu étudiée. De fait, les travaux sur le sujet sont à peu près inexistants. ${ }^{7}$ Pourtant, le grand nombre d'éditions, de traductions et de commentaires, publiés au XVIe siècle, témoigne de la large diffusion de cet ouvrage dont une cinquantaine de manuscrits nous sont parvenus. De 1472 à 1597 , plus d'une trentaine d'éditions complètes ou partielles paraissent, dont trois à Paris chez Josse Bade et une dizaine à Lyon chez Sébastien Gryphe. En 1538, Jean Colin donne une traduction française du Songe de Scipion ${ }^{8}$ et, en 1549 , Juan de Jarava le traduit en espagnol. De son côté, Etienne Dolet imprime en 1540 la traduction grecque que Théodore Gaza avait faite du Somnium Scipionis de Cicéron. Tant à Londres ${ }^{9}$ qu'à Paris, les commentaires abondent. A la suite de Juan Vives, Ramus commente le 
texte et en 1546 fait imprimer ses commentaires qu il réédite en 1550 et dédie au cardinal Charles de Lorraine." Déjà au XVe siècle. François Villon cite Macrobius dans sa Ballade des femmes de Paris et l'auteur des Saturnales est, parmi les écrivains classiques, celui que Pétrarque cite le plus fréquemment."

Alors que la tradition philosophique avait retenu du Commentaire du Songe de Scipion les argu ments relatifs à l'immortalité de l'âme. la tradition littéraire avait. quant à elle. récupéré le motif du songe. On n’a ici qu’à évoquer le fameux Roman de la Rose. ${ }^{2}$ Piers the Plowman, les Contes de Canterbury ou le Songe de Pantagruel de François Habert sur lequel nous reviendrons plus loin. Nous verrons que Rabelais exploite une autredimension. négligée quoique capitale. du Songe de Scipion: le salut que confère la virtus. ${ }^{13}$

Or les commentateurs de loeuvre de Rabelais ignorent ou taisent ce possible renvoi. ${ }^{14}$ Seul M.A. Screech y fait allusion pour rejeter aussitôt cette référence au profit de la définition qu’en aurait donnée Rabelais dans la Briefie declaration: "Macrobe, homme de longue vie."Is Pourtant, que ces annotations soient ou non de la main de Rabelais. l"explication qu'offre la Briefie declaration n'exclut pas le rapprochement avec Macrobius; elle lui donne au contraire plus de poids. En effet, lors de la visite de l'île des Macréons, l'attention du lecteur est constamment sollicitée par le caractère antique des lieux et des habitants. La remarque de Panurge "Macraeon. en grec. signifie vieillart, home qui a des ans beaucoup" $(Q L, 25)$ et les gloses de la Briefie declaration mettent en évidence cette pérennité qui sied aussi à Macrobius dont les oeuvres avaient traversé dix siècles pour parvenir aux lettrés de la Renaissance. ${ }^{16}$

Par ailleurs, d'autres indices plus probants que celui d'une semblable vétusté, fondent notre rapprochement. Premièrement, Rabelais connaît Macrobius dont il cite les Saturnales. ${ }^{17}$ De plus, dès la première apparition de Macrobe au début du chapitre 25, le narrateur précise "(Ainsi nommoient ilz leur maistre eschevin)." Se fondant sur cette spécification, M.A. Screech fait du nom propre "Macrobe" un appellatif désignant le poste qu'occupe le magistrat de l'île des Macréons. ${ }^{18} \mathrm{Il}$ interprète ainsi à contresens la formule rabelaisienne; car il s’agit bien d'un véritable nom propre comme l'indique l'omission de l'article défini dans la présentation du prélat de l'île des Papimanes: "Tant grandes feurent leurs exclamations que Homenaz y accourut (ainsi appellent ilz leur evesque) [ . . ] "( $Q L, 48)$. Toutefois, cette précision quant à la fonction politique qu'assure Macrobe n'est pas dénuée d'intérêt. D'abord, ce titre diffère de ceux que le narrateur attribue aux gouvernants des autres îles: "roy," "potestat," "gouverneur." Il s'apparente surtout au titre de vir consularis sous lequel de nombreuses éditions du Commentaire du Songe de Scipion présentent Ambrosius Aurelius Theodosius Macrobius..$^{19}$ Rabelais met ainsi la puce à l'oreille de ses lecteurs. 
Enfin, une dernière indication, plus déterminante, renforce le rapprochement. Macrobe, personnage fictif du Quart Livre, intervient dans le cadre d'une thématique qui fait écho à celle du Songe de Scipion: l“immortalité qu'acquiert le héros en récompense de ses actions vertueuses au service de la res publica.

Dans le fragment de La République que nous a conservé Macrobius. Scipion l'Africain apparaît en songe à son petit-fils adoptif. Il lui prédit sa destinée terrestre et, pour l'inciter au service de la patrie, lui révèle la récompense réservée à ceux qui se sont dévoués à l'Etat: la jouissance de la vie éternelle (Somm.Scip. 3,I). ${ }^{20}$ Scipion le Jeune, désireux de rejoindre dans la "Voie Lactée" son père Paul Emile et le premier Africain. deux héros par leur valeur militaire et morale, veut alors hâter le moment de sa mort. Mais son grand-père lui rappelle qu'il doit d'abord exercer son âme aux plus hautes activités: celles qui contribuent à la préservation. à la prospérité et à l'accroissementde la patrie (Somm.Scip. 9.2). ${ }^{21}$ Il l'exhorte à la pratique des vertus civiles qui élèvent l'homme et lui ouvrent le chemin de la béatitude éternelle. Dans son commentaire. Macrobius infléchit légèrement cette priorité de l'activité politique sur la philosophie en consacrant l'apothéose des hommes qui ont concilié les vertus contemplatives (virtutes exemplares) et les vertus politiques (virtutes politicae) (Macrobius. Comm. I. 8. 12 et II. 17. 4-13).

On retrouve donc au coeur même du Songe de Scipion les principes éthiques qui doivent guider la conduite de l'homme dans le gouvernement de la république et assurer son séjour parmi les Bienheureux. Le Songe s'achève sur la démonstration de l'immortalité de l'âme, empruntée au Phèdre de Platon, propos qui clôt aussi le chapitre 27 du Quart Livre. Sans parler d’emprunts ou d'influences directes. on ne peut éviter de remarquer certaines similitudes thématiques entre les deux textes. Les mêmes motils éthiques prévalent dans la tempête en mer, l'escale à l'île des Macréons et le récit des morts de Guillaume du Bellay et du Dieu Pan. Alors que la plupart des études exploitent la dimension théologique de ces épisodes. ${ }^{22}$ la reconnaissance de l'homonymie onomastique et les résonnances que trouve dans ces pages le Songe de Scipion suggèrent plutôt une lecture éthique. ${ }^{23}$

Dès le début du Tiers Livre. Panurge, las de la guerre, annonce son intention de se marier afin, dit-il. que "cessent les armes" et qu il puisse "un an pour le moins, respirer de l'art militaire." ( $T L .7)$ Toutefois, les doutes qui lassaillent quant à la fïdélité de sa future épouse ébranlent sa décision et le plongent dans une profonde perplexité. Alìn de résoudre son dilemme. Panurge entreprend, sur le conseil de ses amis, une série de vaines consultations qui se poursuivront tout au long du Tiers Livre. A l'issue de cellesci. il décide de prendre la mer pour obtenir le mot de la Dive Bouteille. Le Quart Livre relate cette navigation ponctuée d’escales dans diverses îles. 


\section{4 / Renaissance and Reformation}

La tempête qui survient au cours de cette pérégrination confronte les voyageurs à la mort et dépeint leurs réactions devant ce péril. Déjà le chapitre précédent avait attiré l'attention du lecteur sur les circonstances entourant le trépas du géant Bringuenarilles. Son étranglement fatal, avec un morceau de beurre frais, avait donné lieu à une longue énumération de morts étranges. La perspective de la mort, question étroitement liée au salut et à l'immortalité de l'âme, constitue le motif central de l'épisode. ${ }^{24}$ C'est ce que font ressortir les doléances répétées de Panurge, ${ }^{25}$ sa remarque au pilote ${ }^{26}$ ainsi que les propos de Gargantua et d'Epistémon relatifs à la mort. ${ }^{27}$

De plus, les premières lignes du chapitre 18 présentent les moyens que Panurge met en oeuvre pour assurer le salut de son âme. Peu avant que n'éclate la Tempête, la flotte de Pantagruel croise un convoi de "neuf orques chargées de moines" en route pour le concile de Chésil. Entrant "en excès de joye," Panurge salue les "beatz peres," recommande le salut de son âme à leurs prières et fait jeter en leurs navires provisions et argent pour les âmes des trépassés. Alors que Panurge interprète cette rencontre comme un présage de bonne fortune, Pantagruel, lui, reste "toutpensif et melancholique" $(Q L, 18)$. Frère Jean s'enquiert des causes d'une "telle fascherie non acoustumée" mais la question reste pendante car se lève un "tyrannicque grain et fortunal nouveau." La suite de cet épisode apportera une réponse aux interrogations de Frère Jean. A l'exemple du Songe de Scipion, elle fera ressortir que la vertu active menée en fonction du bien commun. s’avère la seule voie de salut.

Alors que la tempête fait rage, chacun met la main à l'ouvrage et tous les passagers s’activent pour échapper au danger. Seul Panurge, tremblant de peur, se plaint, se morfond, gémit sur son sort et demeure le témoin passif des efforts que déploient ses compagnons:

Pantagruel [ . . . ] tenoit l'arbre fort et ferme. Frere Jan s'estoit mis en pourpoinct pour secourir les nauchiers. Aussi estoient Epistemon. Ponocrates et les aultres. Panurge restait le cul sus le tillac, pleurant et lamentant. (QL, 19)

Rappelons le jugement que, dans le prologue du Tiers Livre, le narrateur profère à l'endroit des oisifs:

ay imputé à honte plus que mediocre estre veu spectateur ocieux de tant vaillans, disers et chevalereux personnaiges $[\ldots]$ et ne me esvertuer de moy mesmes et non y consommer ce rien, mon tout, qui me restoit.

Panurge incite même les membres de l'équipage à cesser d'agir: "Dea. beaulx amys, puys que surgir ne povons à bon port. mettons nous à la rade. je ne sçay où. Plongez toutes vos ancres. Soyons hors ce dangier, je vous en 
prie. Nostre amé, plongez le scandal et les bolides, de grace." $(Q L, 20)$ Ses recommendations ne sont en fait que des exhortations à l'inaction. ${ }^{28}$ Son oisiveté contraste avec l'ardeur qui anime Frère Jean. Les comportements qu'ils adoptent au cours de l'orage ne sont pas sans rappeler ceux des protagonistes de l'attaque du clos de Seuillé. ${ }^{29}$ Au chapitre 23 du Quart Livre, Frère Jean remémore le sac du clos de Seuillé. Avec la même vaillance, il avait alors secouru la vigne et combattu les pillards de Lerné qui comme Panurge se vouaient à tous les saints. Celui-ci réitère d'ailleurs leurs supplications: "Confession! Confession! Confiteor! Miserere! In manus!" (Gargantua, 25) Quant à l'inertie de Panurge, elle évoque la passivité des moines. Le souhait qu'il émet au plus fort de la tempête renforce ce parallèle: "Pleust à la digne vertus de Dieu que à heure praesente je feusse dedans le clos de Seuillé [... ]" $Q L, 20)$.

En désespoir de cause, Panurge manifeste le désir de faire son testament. Frère Jean réplique aussitôt: "Vertus Dieu! parle tu de testament à ceste heure que sommes en dangier, et qu'il nous convient evertuer ou jamais plus?" $(Q L, 19)$. Répondant à la même requête, Epistémon reprend cette . formule qui deviendra un leitmotiv dans la suite du récit. Il établit alors une comparaison entre la nécessité de s’évertuer pour échapper au naufrage et l'obligation de déployer toutes ses énergies au combat. Il accentue ainsi le parallèle entre la tempête, ce "tyrannicque grain," et laguerre, parallèle que laissaient déjà pressentir les réminiscences du sac du clos de Seuillé:

Faire testament, dist Epistemon, à ceste heure qu'il nous convient evertuer et secourir nostre chorme sus poine de faire naufraige, me semble acte autant importun et mal à propous comme celluy des Lances pesades et mignons de Caesar entrant en Gaule, les quelz se amusoient à faire testamens et codicilles, lamentoient leurs fortune, pleuroient l'absence de leurs femmes et amys Romains, lorsque, par necessité, leurs convenoit courir aux armes et soy evertuer contre Ariovistus leur ennemy. ( $Q L$, 21)

Al'issue de la Tempête, Epistémon renouvelle cette comparaison guerrière ${ }^{30}$ en citant les paroles du consul C. Flaminius et de Caton d'Utique: "Par force et vertus il nous convient evader, et à fil d'espée chemin faire par le mylieu des ennemis." $(Q L, 23)$

L'expression "s'évertuer" réapparaît aussi dans les propos du géant: "Si, dist Pantagruel, paour il [Panurge] a eu durant ce colle horrible et perilleux fortunal, pourveu que au reste il se feust evertué, je ne l'en estime un pelet moins." $(Q L, 22)$ (C'est nous qui soulignons.) Epistémon l'emploie encore à deux reprises dans son argumentation:

Pourtant, icelluy [Dieu] fault incessamment implorer, invocquer, prier, requerir, supplier. Mais là ne fault faire but et bourne: de nostre part, convient 
pareillement nous evertuer, et. comme dict le Sainct Envoyé. estre cooperateurs avecques luy. [ . . . En veiglant. tavaillant |sic|, soy evernumt, toutes choses succedent à soubhayt et bon port. (QL. 23) (C'est nous qui soulignons.)

Le verbe "s"évertuer" constitue donc le mot-clé de lépisode. De plus, il appert qu'outre les connotations religieuses qu"on lui prête. le mot "s ésvertuer" ressortit à l'activité civile (guerre, bien commun de la cité) impliquant l'exercice d'une vertu toute pratique. Il fait référence à l'action vertueuse que l'homme doit mener dans la conduite des affaires humaines, au rôle décisif qu il doit assumer. Ce leitmotiv fait écho aux conseils que Scipion 1'Africain prodigue à son petit-fils (Somm. Scip. 2. 23-24: 2, 31-33). "S"évertuer" doit donc être entendu comme lapplication des vertus que Mac robius énumère dans son Commentaire: prudence, justice, force et tempérance ${ }^{31}$ (Comm, I, 1,8; I, 8).

Tant dans le Songe de Scipion que dans le récit de la Tempête, ces vertus cardinales se révèlent la condition du salut, dans la double acception de ce terme. Au sens propre, les navigateurs, en s'évertuant, échappent à la morte. Au sens figuré, le préalable requis pour que l’âme, après la mort. accède dans laudelà à un état de félicité éternelle réside aussi dans cet acte. ${ }^{32}$ L'escale à l'île des Macréons mettra en relief l'ambivalence de ce terme, pierre de touche de l'immortalité.

L'orage passé, l'équipage a survécu au péril du naufrage. Pantagruel annonce que la terre est en vue et bientôt Epistémon s’écrie: "Haye. haye.je voy terre, je voy port. je voy grand nombre de gens sus le havre." $(Q L .22)$ Les Macréons vont à la rencontre des voyageurs qui débarquent enfín dans l'île où Macrobe les accueille. La conversation s'engage et les discussions portent sur la mort des Démons et des Héros exemplifiée par le souvenir du décès de Guillaume du Bellay. La structure de ce deuxième épisode actualise en fait une métaphore empruntée à Plutarque (Du génie de Socrate. 593f) et développée dans le chapitre 21 du Tiers Livre. Ce passage précédait la consultation du vieux poète Raminagrobis, à l’article de la mort. Dans ce texte, les marins et les voyageurs "dedans leurs naufz en haulte mer" s'apparentent aux passagers de la Thalamège. Ils sont comparés aux humains qui savancent parmi "les troubles et sollicitudes terrienes." Lorsqu ils approchent du havre, les gens les reçoivent par des gestes et des paroles. comme les Macréons l'avaient fait à l'égard de la compagnie pantagruéline. De la même façon, "les Anges, les Heroes, les bons Daemons" accueillent "les humains prochains de mort, comme de port tresceur et salutaire, port de repous et de tranquilité." $(T L, 21)$ Dans les deux textes, suit la même allusion au décès de Guillau me du Bellay. Microcosme de l'escale à l'île des Macréons, ce passage du Tiers Livre confirme la double signification du salut dont nous avons fait état précédemment. 
Ainsi, alors que le premier volet de la trilogie (la Tempête) démontrait que le seul chemin pouvant conduire au salut, ici-bas et dans l'au-delà, était de s'évertuer, le deuxième épisode en fournit le modèle. En effet, dans les chapitres 26 et 27 du Quart Livre, le rappel de la mort du seigneur de Langey illustre cette thèse exposée dans le Songe de Scipion.

Peu après son arrivée dans l'île, Pantagruel s'enquiert auprès du vieux Macrobe des causes de la tempête. Celui-ci explique qu'au moment de la mort des Daimons et des Héros (ces termes étant presque synonymes) surviennent des désordres naturels:

Au trespas d'un chascun d'iceulx, ordinairement oyons nous par la forest grandes et pitoyables lamentations, et voyons en terre pestes, vimeres et afflictions; en l'air, troublemens et tenebres; en mer, tempeste et fortunal. $(Q L, 26)$

Pantagruel confirme la véracité des dires de Macrobe mais il précise que la "discession" des âmes nobles et insignes s'accompagne de troubles d'ordre civil: "lamentations des peuples, mutations des religions, transpors des Royaulmes, et eversions des Republicques." $(Q L, 26)$ Epistémon appuie ces propos en donnant une première fois l'exemple du "decés du preux et docte chevalier Guillaume du Bellay [ . . ]." $(Q L, 26)$ Il insiste sur le rôle politique de ce serviteur de l'Etat ${ }^{33}$ et y reviendra au chapitre suivant. Pantagruel renchérit en évoquant son antithèse: Hérode qui avait usé d'artifices frauduleux afin de laisser croire aux étrangers quà son trépas, fin d'un règne tyrannique, quelqu'âme héroïque était décédée. Puis il cite Néron et Tibère.

C'est donc l'exercice du bon et juste gouvernement de l'homme et de la cité qui rend les âmes "nobles, precieuses et Hroïcques." Guillaume du Bellay accède à ce rang par les qualités éthiques qu il a manifestées au cours de son existence. Pantagruel accentue cette idée en faisant de ces âmes les réceptacles de tout savoir "touchant le bien et utilité publicque ou privée" $(Q L, 27)$. Une question de Frère Jean amène enfin Pantagruel à conclure que toutes les âmes intellectives sont immortelles. Ce deuxième épisode se termine donc, à l'instar du Songe de Scipion, sur l'affirmation de l'immortalité de l'âme. Les chapitres 25,26 et 27 tendent à faire valoir que les hommes de bien, parmi lesquels se range le docte et preux Chevalier de Langey, garantissent leur propre salut en contribuant par leurs actions vertueuses à celui de la patrie, ainsi que l'invoque le premier Africain pour raffermir la volonté du jeune Scipion.

Dans le dernier volet de ce triptyque, soit le chapitre 28 du Quart Livre, Pantagruel narre une histoire concernant le "trespas des Heroes." Il relate l'annonce de la mort du Dieu Pan dans lequel il reconnaît le "grand Servateur des fideles." Ce chapitre a fait couler beaucoup d'encre. ${ }^{34}$ Parmi les multiples sources d'inspiration relevées par les commentateurs, un texte, 


\section{8 / Renaissance and Reformation}

le Songe de Pantagruel de François Habert, offre, dans la perspective que nous avons développée jusqu'à maintenant, un intérêt tout particulier. En premier lieu, la préfiguration du Christ sous les traits de Pan, le grand Dieu, se trouve également dans ce poème publié en $1542 .{ }^{35}$ Mais, fait plus notable encore, cet écrit présente de nombreux points de ressemblance avec le Songe de Scipion. ${ }^{36}$

Le texte de Habert emprunte d'abord la même structure narrative. Les deux récits s'ouvrent sur un banquet. Le souvenir des discussions imprègne si fortement l'esprit du personnagenarrateur que, la nuit venue, Pantagruel voit apparaitre en rêve son père Gargantua. Cette brève introduction du Songe de Pantagruel mime la courte mise en scène du fragment de Macrobius. Gargantua indique à son fils le sentier où il doit cheminer afin que son âme parvienne "droict es cieulx": il doit se consacrer ici-bas à la tâche du berger (Songe de Pantagruel, v. 215-216) et veiller sur le troupeau du grand Dieu Pan. Puis intervient un troisième personnage, Panurge, qui s'éclipse bientôt, comme dans le Songe de Scipion l'apparition de Paul Emile ne constituait qu'un interlude. Les similitudes que présentent les deux songes sont frappantes. Malgré ses colorations évangéliques, le contenu du Songe de Pantagruel partage la visée protreptique du Songe de Scipion et rejoint l'apologie rabelaisienne de l'Homme engagé sur la voie de la virtus. L'auteur du Quart Livre ne devait certes pas ignorer l'existence de ce texte qui met en présence trois personnages empruntés à la geste de Rabelais. ${ }^{37}$

Le témoignage que nous livre Pantagruel, dans le chapitre 28 du Quart Livre, fonctionne, nous semble-t-il, comme la confirmation "exemplaire" des propos tenus à l'endroit de Guillaume du Bellay. La mort des héros et, a fortiori, celle du Christ, parangon de toutes vertus, s'accompagnent de prodiges par lesquels les cieux manifestent aux hommes l'envol de ces âmes. Le chapitre 28 couronne la trilogie par la mention du modèle idéal: l'archétype du dieu fait homme qui, assurant son salut et sa propre immortalité par la mort, assure ultimement celui de toute l'humanité.

La logique de l'enchaînement des trois épisodes suit une progression ascendante. Au cours de la Tempête, tous les voyageurs s'évertuent pour échapper au naufrage, à l'exception de Panurge qui, rappelons-le, ne veut plus aller en guerre et s'adonne à l'économie mercantile du salut. Pantagruel, Epistémon et Frère Jean se portent au secours des marins et les hommes parviennent finalement sains et saufs à l'île des Macréons, havre de salut, séjour des Héros et des Daimons. Le deuxième épisode met de l'avant les "fins" d'une âme hérö̈que "tant nécessaire à la gloire et à la protection de la France." L'exemple de la mort de Guillaume du Bellay, ce héros, ce semi-dieu, fait ressortir que la finalité de l'homme dans l'au-delà est déterminée par sa participation à la res publica. Son aspiration est certes individuelle mais toute empreinte d'un humanisme civique. Le passage 
final constitue le dernier palier de cette ascension. Il fournit l'exemple-type du "Sauveur." La mort du Christ, évoquée dans ces pages, complémente la théorie du salut que se méritent les héros par la pratique des vertus civiles. Elle ouvre à l'activité vertueuse un champ de réalisation plus large que le seul domaine politique.

Ce triptyque pose donc, avec une particulière intensité éthique, le problème du salut et celui des moyens qui doivent être mis en oeuvre pour l'assurer. Syncrétique, la pensée rabelaisienne allie à un synergisme évident l'idée centrale de la virtus antique sur laquelle doit se fonder la collaboration de l'homme aux desseins divins. L'inscription du nom de Macrobe au coeur de cette trilogie et les résonnances éthiques qu'y trouve le Songe de Scipion justifient, à notre avis, cette synthèse.

\section{Université de Montréal}

Notes

1 Nous utilisons les éditions suivantes des oeuvres de François Rabelais: Gargantua, éd. R. Calder et M.A. Screech (Genève-Paris: Droz-Minard, 1970); Le Tiers Livre, éd. M.A. Screech (Paris-Genève: Droz, 1964); Le Quart Livre, éd. R. Marichal (Genève: Droz, 1947).

2 On compte sept occurrences du nom "Macrobe" dans les chapitres étudiés: $Q L$, chap. 25, p. 127 (2) et p. 128 (1); titre du chap. 26 (1); chap. 26, p. 129 (1); chap. 27, p. 132 (1) et p. 135 (1).

3 Je suis tout particulièrement reconnaissante au Professeur GuyH. Allard qui a porté ce renvoi à mon attention.

4 Afin d'éviter toute ambiguité, nous emploierons "Macrobius" pour désigner l'auteur des Saturnales et "Macrobe" lorsqu'il sera question du personnage fictif du Quart Livre.

5 J.H.M. Salmon, "Cicero and Tacitus in Sixteenth-Century France," The American Historical Review, 85 (1980), 316. Angelo Mai devait découvrir le reste de l'ouvrage en 1820.

6 Macrobius, Commentary on the Dream of Scipio, translated withan introduction and notes by W.H. Stahl, (New York: Columbia University Press, 1952); W.H. Stahl, "Dominant Traditions in Early Medieval Latin Science," Isis 50 (1959), 95-124; P.M. Schedler, Die Philosophie des Macrobius und ihr Einfluss auf die Wissenschaft des christlichen Mittelalters (Münster, 1916); P. Courcelle, "La postérité chrétienne du Songe de Scipion," Revue des études latines 36(1958), 205-234; E. Jeauneau, "Macrobe, source du platonisme chartrain," Studi Medievali I (1960), 3-24; H. Silvestre, "Note sur la survie de Macrobe au moyen âge," Classica et mediaevalia 24 (1963), 170-180; J. Flamant, Macrobe et le néo-platonisme latin, à la fin du IVe siècle (Leiden: Brill, 1977).

7 On s'est surtout intéressé à l'influence de Macrobe sur la littérature espagnole: D. BakerSmith, "Juan Vives and the Somnium Scipionis," in Classical Influences on European Culture A.D. 15001700 (Cambridge: Cambridge University Press, 1976), pp. 239-244; L.J. Swift, "Somnium Vivis y el Sueno de Espicion," in Homenaje a Luis Vives (Madrid,1977), pp. 89-112; M.D. McGaha, "The Influence of Macrobius on Cervantes," Revue de littérature comparée 4 (1979), 462-469. Qúant à C.R. Ligota, il propose une analyse comparative de la conception du soleil chez Macrobe et quelques penseurs italiens: Ficin, Palmieri, etc., voir "L'influence de Macrobe pendant la Renaissance," in Le soleil à la Renaissance. Sciences et mythes (Bruxelles-Paris: Presses universitaires de Bruxelles-Presses universitaires de France, 1965), pp. 463-482.

8 Jean Colin,Lesonge de Scipio, extraict du sixiesme livre de la République de M.T. Cicero (Paris, 1538), $14 \mathrm{ff}$.

9 Florentius Volusenus, Scholia Seu Commentariorum Epitome in Scipionis Somnium (Londres: Robert Redman,[1534]). 


\section{0 / Renaissance and Reformation}

10 Somnium Scipionis, ex sexto libro de republica M. Tullii Ciceronis, Perri Rami Veromandue praelectionibus explicatum (Paris, 1546); Scipionis Somnium, ex sexto M. T. Ciceronis de republica, P.R. Praelectionibus explicatum, secunda editio (Paris: M. David, 1550), 74 p..

11 Concernant l'influence de Macrobius sur Pétrarque, voir Pierre de Nolhac. Pétrarque et I'humanisme, t. Il (Paris: Honoré Champion, 1965), p. 103.

12 Guillaume de Lorris et Jean de Meun, Le Roman de la Rose, éd. F. Lecoy (Paris: Champion, 1966). II. 6-10. Sur l'utilisation du Songe dans le Roman de la Rose, voir E. Langlois, Origines et sourres du Roman de la Rose (Paris, 1891).

13 Au mot "vertu" lourd de connotations moralisatrices, nous préférons substituer le terme "virtus" dont Macrobius établit la nomenclature dans son Commentaire $(\mathbf{I}, 8)$.

14 Les divers éditeurs des textes rabelaisiens (A. Lefranc, J. Boulenger, R. Marichal, G. Demerson) renvoient simplement le lecteur à la définition de la Briefve declaration. Aucun d'eux ne mentionne la référence possible à Macrobius.

15 M.A. Screech. "The Death of Pan and the Death of Heroes in the Fourth Book of Rabelais. A Study in Syncretism," BHR 17 (1955), 48, n.1. Dans son Rabelais (London: Duckworth, 1979), il passe sous silence cette allusion et n'a plus la même certitude quant à la paternité de la Briefve declaration. Il écrit à la page 369de son ouvrage: "Whoever compiled the Briefve declaration [... ]."

16 C'est du moins l'impression qui se dégage à la lecture de la page-titre de plusieurs éditions du Commentaire du Songe de Scipion publiées au XVIe siècle: Macrobii Ambrosii Aurelii Theodosii In Somnium Scipionis, Lib. II. Saturnaliorum, Lib. VII. Ex varijs, ac vetustissimis codicibus recogniti, \& aucti. Lugduni. Apud Seb. Gryphium. (1542, 1548, 1550). (C'est nous qui soulignons.)

17 A la fin du chapitre 3 du Gargantua. Le nom de Macrobius apparaît aussi dans le chapitre $42 b$ du Cinquiesme Livre. Par ailleurs, l'auteur de la Briefve declaration connait le Songe de Scipion dont il mentionne le titre dans la définition des "Catadupes du Nil," p. 279.

18 M.A. Screech, Rabelais, p. 352.

19 Macrobii aurelii theodosii viri consularis, In Somnium Scipionis Libri II, Saturnaliorum Libri VII. Nunc denuo recogniti \& multis in locis aucti ... Lugduni, Seb. Gryphium, 1538. (C'est nous qui soulignons.) Dans son Dictionnaire de la langue française du XVle siècle, E. Huguet donne sous l"entrée "consul" le synonyme "échevin" et précise que ce titre s'applique aux magistrats des villes antiques.

20 "Sed quo sis, Africane, alacrior ad tutandam rem publicam, sic habeto: omnibus qui patriam conservaverint adiuverint auxerint, certum esse in caelo definitum locum ubi beati aevo sempiterno fruantur." Ambrosii Theodosii Macrobii Commentarii in Somnium Scipionis, éd. J. Willis (Leipzig: Teubner, 1963), II, 157.

21 "Hanc tu exerce optimis in rebus. sunt autem optimae curae de salute patriae." Ibid., p. 163.

22 M.A. Screech a bien fait voir les implications théologiques de la tempête dans son Rabelais, p. 345 et dans "L'Evangélisme de Rabelais. Aspects de la satire religieuse au XVIe siècle," $E R$ II (1959), 43. Comme le note Cholakian dans "A Reexamination of the Tempest Scene in the Quart Livre," French Studies 21 (1967), 104110 et surtout p. 105, il est certain que l’épisode de la tempête n'est pas étranger aux querelles qui opposaient les théologiens de l'époque sur les problèmes du libre arbitre et de la grâce divine. Mais contrairement à ces études qui confinent le propos à des considérations théologiques, il nous semble que des préoccupations éthiques traversent l'ensemble de ces chapitres et en fondent l'articulation centrale.

23 Il faut veiller à ne pas confondre éthique et morale religieuse. L'éthique, au sens o nous l'entendons, désigne toute espèce d’agir humain subordonné au bien commun.

24 Dans le songe de Scipion, dès son apparition, le premier Africain annonce au héros le moment de sa mort et les conditions de son salut (Somm.Scip.,I, 2-3).

25 "Je naye, je naye, je meurs. Bonne gens, je naye." $(Q L, 18)$; "Estoit ce icy que de perir nous estoit praedestiné? Holos, bonnes gens, je naye, je meurs Consummatum est. C'est faict de moy." $(Q L, 19)$. 
26 " $[\ldots]$ nous sommes doncques continuellement à deux doigtz près de la mort" $(Q L, 23)$.

27 "Ores, si chose est en ceste vie à craindre, après l'offense deDieu, je ne veulx dire que soit la mort. Je ne veulx entrer en la dispute de Socrates et des Academicques: mort n'estre de soy maulvaise, mort n'estre de soy à craindre. Je diz ceste espece de mort par naufraige estre, ou rien n'estre à craindre. Car, comme est la sentence de Homere, chose griefve, abhorrente et denaturée est perir en mer." $(Q L, 22)$; "Je consydere que si vrayement mourir est (comme est) de necessité fatale et inevitable, en telle ou telle heure, en telle ou telle façon mourir est en la saincte volunté de Dieu." $(Q L, 23)$.

28 R. Cholakian a mis en lumière cet aspect du comportement de Panurge. Cf. op.cit., p. 106.

29 M.A. Screech dans "L'Evangélisme de Rabelais. Aspects de la satire religieuse au XVIe siècle," p. 42 de même que D. Coleman, dans "Rabelais: two versions of the "Storm at Sea" Episode," French Studies, 23 (1969), 118, ont relevé le parallélisme de ces deux épisodes.

30 Comme le souligne G. Demerson à propos des comparaisons animales (Rabelais [Paris: Balland, 1986], p. 28 et p. 141), les métaphores ne sont jamais innocentes chez Rabelais.

31 Panurge mentionnait ces quatre vertus cardinales dans l'Eloge des dettes du Tiers Livre $(T L, 2)$.

32 Nous verrons dans un article ultérieur que l'au-delà métaphysique intéresse moins Rabelais que les moyens mis en oeuvre pour y accéder.

33 " [... l lequel vivant, France estoit en telle felicité que tout le monde avoit sus elle envie, tout le monde se y rallioit, tout le monde la redoubtoit. Soubdain après son trespas, elle a esté en mespris de tout le monde bien longuement." $(Q L, 26)$.

34 Notons, entre autres, la remarquable contribution de M.A. Screech à l'intelligence théologique de cet épisode: "The Death of Pan and the Death of Heroes in the Fourth Book of Rabelais. A Study in Syncretism," pp. 36-55; A.J. Krailsheimer a aussi consacré plusieurs articles à ce chapitre: "Rabelais and the Pan Legend,"French Studies 2(1948), 158-161; "Rabelais et Postel," BHR 13 (1951), 187-190; "The Significance of the Pan Legend in Rabelais Thought," Modern Language Review 56 (1961).

35 Ont signalé cette correspondance textuelle: A. Hulubei, dans L'Eglogue en Franceau XVle siècle. (1515-1589), (Paris, 1938), note 3, p. 237 et M.A. Screech dans "The Death of Pan and the Death of Heroes in the Fourth Book of Rabelais. A Study in Syncretism," p. 46 ainsi qu'à la page 359 de son Rabelais.

36 Dans l'introduction de sa récente édition du Songe de Pantagruel (pp. 112-115), J. Lewis trace quelques parallèles entre ces deux textes. Voir François Habert, Le Songe de Pantagruel, published with an introduction by John Lewis, ER 18 (1985) 103-162.

37 A. Lefranc a mis en relief l'influence du Songe de Pantagruel sur le Tiers Livre de Rabelais. Voir Lesoeuvres de Francois Rabelais, édition critique publiée sous la direction d'Abel Lefranc (Paris, 1912-1931), vol. V, pp. LVII-LXIV, et J. Lewis, op. cit., Introduction, pp. 115-118. 